\title{
Protective effect of Ellipticine in ovalbumin (OVA)-induced murine model of allergic rhinitis via dual inhibition of COX-2 and NF-KB
}

\author{
Jian Wang ${ }^{a}$, Xiao Liu ${ }^{a}$, Zhi Liu ${ }^{a}$, Yanxia Ge ${ }^{b}$ and Shuangwen $\mathrm{He}^{\mathrm{a}}$ \\ a'Department of Otorhinolaryngology Head and Neck Surgery, Xi'an International Medical Center Hospital, Xi'an, 710100, China; \\ ${ }^{b}$ Department of Otorhinolaryngology Head and Neck Surgery, Xijing Hospital, Xi'an, 710032, China
}

\begin{abstract}
Allergic rhinitis (AR) is a serious health concern across the globe. Despite its non-fatal character, it accounts for affecting millions of people across the world and is deemed responsible to affect their quality of life and put a significant economic burden. In the current study, we aimed to assess the anti-allergic and anti-inflammatory effects and the underlying molecular mechanisms of ellipticine (ETC) against AR using ovalbumin (OVA)-induced murine model of allergic rhinitis. The ETC was administered to mice via intra-peritoneal route after suspending in $5 \% \mathrm{CMC}$ after sensitization by OVA. Results of the study suggested that ETC causes a significant reduction the nose rubs as compared to disease control. A significant reduction in the serum level of histamine, IgG1, TNF- $a_{\text {, }}$ IL-1 $\beta$, MIP-2, and IL- 6 was found in ETC treated group in a dose-dependent manner as compared to OVA challenged mice. It also reduces eosinophils in BALF of AR mice. In western blot analysis, the expression of aberrantly activated COX-2 and NF-KB found significantly reduced in ETC treated group due to inhibition of TLR- 4 and caspase- 1 as compared to disease-control mice. ETC showed significant interaction with residues of the active site of COX-2 and NF-KB. Collectively, our results indicated that ETC can be used to improve present therapeutic strategies against AR.
\end{abstract}

\section{ARTICLE HISTORY}

Received 25 November 2020

Accepted 14 February 2021

\section{KEYWORDS}

allergic rhinitis;

inflammation; cytokines;

COX-2; NF-kB

\section{Introduction}

Allergic rhinitis is a serious global health concern affecting mucosal tissue of the nasal cavity. Despite its non-fatal character, it accounts for affecting millions of people across the world and is deemed responsible to affect their quality of life and put a significant economic burden. The AR is caused due to allergens, which are responsible for the induction of hypersensitivity reaction in the affected individuals. The allergens are any chemical or agent found indoor or outdoor, such as dust, mites, pollens, paints, molds, etc. to induce a cascade of inflammatory reactions mediated via immunoglobulins E (IgE) like antibodies (Incorvaia et al., 2018; Small et al., 2018). The clinical symptoms of AR include sneezing, rhinorrhea, itching induced due to acute activation and infiltration of inflammatory cells, which include, mast cells, eosinophils, and other types. These inflammatory cells upon recruitment lead to the production of various inflammatory mediators such as histamine, serotonin, nucleotides, proteases, and tumor necrosis factor (TNF)-a. The inflammation of airway or nasal mucosa, blood vessel enlargement, enhanced secretions, and more importantly, bronchospasm are some of the characteristic features of AR (Bousquet et al., 2019; Brożek et al., 2017). The nasal mucosa is the primary site for allergen exposure and the inflammatory reactions that cause AR symptoms. The mechanisms driving AR pathophysiology are multifaceted and include activation and migration of effector cells, the release of mediators, chemokines, and cytokines from inflammatory cells, and damage to the nasal epithelium and nerve endings (Amin, 2012; Zissler et al., 2016).

The current clinical practice to manage AR is largely dependent upon the use of antihistamines, corticosteroids, and mast cell stabilizers or their combinations (Okubo et al., 2017; Seidman et al., 2015). However, these drugs are associated with serious unwanted effects, such as, mouth dryness, vision blurring, constipation, urinary retention, tachycardia, and sedation which compromise their clinical utility.

CONTACT Shuangwen He enthsw@sina.com 
In the past few decades, the compounds derived from plants or natural products have shown a significant rise as a potential therapeutics against AR. These plant-derived products can stimulate either innate or adaptive immunity by targeting mediators such as lgE, interleukins (IL), prostaglandins, cycloxygenase (COX), lipoxygenase (LIPO), histamine, NF-кB, MCP-1, etc. (Newman and Cragg, 2016; Patridge et al., 2016). Ellipticine (ETC), an alkaloid isolated from the leaves of Ochrosia elliptica and other Apocynaceae plants, is the prototype of intercalators based on the pyridocarbazole system. It has potent anticancer properties, and several of its derivatives have been the subject of clinical trials (Masood et al., 2013; Pandrangi et al., 2014; Stiborova and Frei, 2014; Tao et al., 2017). It showed a significant anti-inflammatory effect in lipopolysaccharide-activated macrophages by targeting the JNK/ AP-1 signaling pathway (Tian et al., 2020). Recently, it showed to block the synergistic effects of IL-17A and TNF-a in epithelial cells and alleviates severe acute pancreatitis-associated acute lung injury in rats ( $\mathrm{Li}$ et al., 2020). Considering the anti-inflammatory effect of ETC on macrophages and acute lung injury, the role of ETC in modulating AR remains unknown. Therefore, the present study was conducted to determine antiallergic effects of ETC in experimentally induced allergy in mice by well-established ovalbumin (OVA)-induced murine model of allergic rhinitis model.

\section{Material and methods}

\section{Chemicals}

Ellipticine together with other chemicals unless otherwise stated were procured from Sigma Aldrich, USA.

\section{Induction of allergic rhinitis in mice using ovalbumin (OVA)}

Fifty-female BALB/c mice, six-week-old were procured from the central animal house of the institute and housed in strict hygienic conditions with an ad libitum supply of food and water. They were evenly distributed in five groups containing 10 animals each group. The animal procedure has been approved by the institutional animal ethical committee for biomedical experiments. The mice were sensitized i.p. with $100 \mu \mathrm{g}$ of emulsified OVA and $20 \mathrm{mg}$ of aluminum hydroxide $\left(\mathrm{Al}(\mathrm{OH})_{3}\right.$, in $100 \mu \mathrm{l}$ of PBS on days 1, 5, and 14. Afterward, the OVA $1.5 \mathrm{mg}$ in $2 \mu \mathrm{l}$ of phosphate buffer saline
(PBS) was instilled in the nasal cavity to induce local immunization once a day from 15 days to day 24. For the OVA-unsensitized mice group, mice were treated i.p. with PBS on days 1,5, and 14 and instilled PBS into the nasal cavity once a day from day 15 to day 24 . The mice were administered orally with distilled water (DW) or ellipticine (ETC) at a dose of 5, 10, and $15 \mathrm{mg} /$ $\mathrm{kg}$ into the stomach once a day for 10 days after suspending in a carboxymethylcellulose (CMC, 5\%) $1 \mathrm{~h}$ before intranasal OVA challenge. The blood and nasal tissue samples were collected on day 24 after anesthesia. The nose-rub that occurred in $10 \mathrm{~min}$ after OVA intranasal administration was counted from day 21 to 23 (three times), where one point was assigned to every five fast, consecutive rubbing movements or to one isolated, relatively slow rubbing movement, as well as to one sneezing movement (Aswar et al., 2015; Yasar et al., 2016).

\section{Histamine assay}

Histamine levels in serum of AR mice were determined using ophthalaldehyde spectrofluorometric analysis as per the previously reported procedure (Han et al., 2016).

\section{Collection of Bronchoalveolar lavage fluid (BALF)}

To perform BALF, animals were anesthetized and a 23-gauge cannula was inserted into the trachea. $1 \mathrm{ml}$ of ice-cold PBS was instilled with a syringe and recovered by gentle aspiration. The recovered BALF was centrifuged ( $150 \times \mathrm{g}$ at $4^{\circ} \mathrm{C}$ for 5 minutes) and the supernatant was recovered for the determination of cytokines in the BALF. The cell pellet was resuspended in $0.5 \mathrm{ml}$ ice-cold PBS and used for further biochemical estimations.

\section{Hematoxylin and eosin (H and E) staining}

Mice were sacrificed after the last dose. Following the collection of blood and BAL, the lung tissues were removed, fixed in $7 \%$ neutral-buffered formalin solution, embedded in paraffin, cut in $3 \mu \mathrm{m}$ sections, and stained with Hematoxylin \& Eosin (H\&E) for examining. The sections were assessed for vascular proliferation, inflammatory cell infiltration, eosinophil infiltration, and the degree of hypertrophy in chondrocytes. The change in each parameter was scored as 0 , no change; 1 , mild change; 2 , moderate change; or 3 , severe 
change. All histomorphological analyses were performed by two histologists with no prior knowledge of the treatment groups (Avincsal et al., 2014).

\section{Preparation of nasal mucosa tissue proteins}

Nasal mucosa tissue was homogenized in homogenization buffer $(20 \mathrm{mM}$ HEPES, $1.5 \mathrm{mM} \mathrm{MgCl} 2,0.2 \mathrm{mM}$ EDTA, $0.1 \mathrm{M} \mathrm{NaCl}, 0.2 \mathrm{mM}$ DTT, $0.5 \mathrm{mM} \mathrm{Na}_{3} \mathrm{PO}_{4}, 1 \%$ protease inhibitor cocktail), then centrifuged at $15,000 \times \mathrm{g}$ for $30 \mathrm{~min}$ at $4^{\circ} \mathrm{C}$. The resulting supernatant was subsequently used for enzyme-linked immunosorbent assay (ELISA), and western blotting.

\section{ELISA analysis}

The levels of thymic stromal lymphopoietin (TSLP), Interleukin 1 beta (IL-1 $\beta$ ), IL-6, Macrophage-inflammatory protein 2 (MIP-2), and TNF- $\alpha$ in the supernatants of homogenized nasal tissues, as well as the level of $\operatorname{lgE}$, $\lg G 1$, and $\lg \mathrm{G} 2 \mathrm{a}$ in the serum of mice, were evaluated by ELISA as per previously reported procedure (Chen et al., 2011; Kamm et al., 2010).

\section{Western blot analysis}

Total nasal mucosa proteins obtained from nasal epithelial cells were extracted from mice using an M-PER Mammalian Protein Extraction Reagent (ThermoFisher Scientific, Inc.) according to the manufacturer's protocol. Protein concentrations were measured via a BCA kit according to the manufacturer's protocol. Isolated proteins were loaded on $10 \%$ SDS-PAGE to separate the proteins and transferred on PVDF membranes (Millipore, Bedford, MA, USA). The membrane was incubated with primary antibodies (1:1000; CST or Abcam, USA) after blocking overnight. The corresponding secondary antibodies (1:2000; Abcam, USA) were further incubated for $2 \mathrm{~h}$. The protein bands were observed using enhanced chemiluminescence substrate (Millipore, Billerica, MA, USA) and photographed with Image Quant LAS 4000 (GE, USA), and were analyzed by Image J software (NIH, Bethesda, MD, USA).

\section{Docking study}

In the present study, CDOCKER protocol inbuilt in Discovery Studio 3.0 was selected to perform docking simulation of ETC with 3D crystal structure COX-2 co-crystallized with Celecoxib (pdb: 3LN1) and NF-kB $\mathrm{p}-50$ homodimer (pdb: 1NFK) as a receptor for docking study. The protein structures were uploaded into the software, water molecules were removed, minimized using CHARMm forcefield, alternate conformers and co-crystallized ligand was removed, and the lost atoms such as hydrogen were added to construct an initial receptor structure for docking. The active site was defined automatically using the "find active site protocol" of the software and selected for docking of ETC. The water molecule and co-crystallized ligand were removed, and the lost atoms such as hydrogen were added to construct an initial receptor structure for docking. The docking was performed using the default parameters of the CDOCKER protocol (Srivastava et al., 2015).

\section{Statistical analysis}

Results were analyzed using the Graphpad Prism software and expressed as the mean \pm standard error of the deviation (S.D.). Statistical analysis consisted of an independent $t$-test and ANOVA followed by Tukey's post hoc test. The results were considered significant at a value of $\mathrm{P}<0.05$.

\section{Results}

\section{Effect of ETC on the symptoms and mediators of OVA-induced AR mice}

Initially, the effect of ETC has been determined after induction of AR in mice. As shown in Fig $1 A$, the frequency of nose-rubs found increased in OVA-sensitized mice with no-treatment, as compared to the sham. However, after administration of ETC, the frequency of nose-rubs decreased significantly in a dose-dependent manner. The effect of ETC was next investigated on various biochemical markers shown to play a critical role in the progression of AR. The serum level of histamine was found significantly upregulated in OVA mice as compared to control, which was reduced significantly upon administration of ETC, Fig 1B. The effect of ETC was also determined on the serum levels of immunoglobulins, such as, IgE, lgG1, and IgG2a which play a key role in mediating immune response against AR. The ETC treated group showed a significant reduction in the serum level of IgE and IgG1, Fig. 1C and D, respectively. Moreover, the level of IgG2a was found significantly increased in ETC treated group as compared to OVA challenged mice, Fig. 1E. These observations suggest that ETC might provide relief against AR 


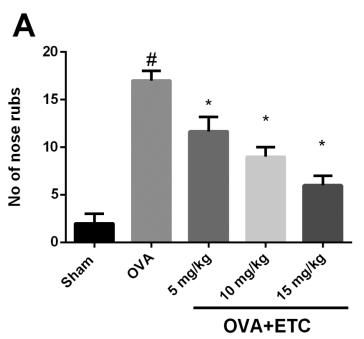

D

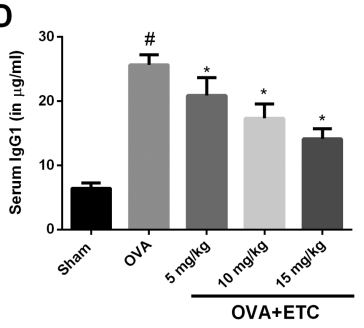

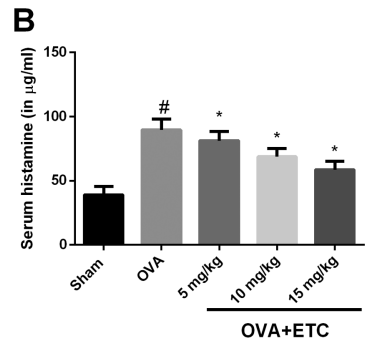

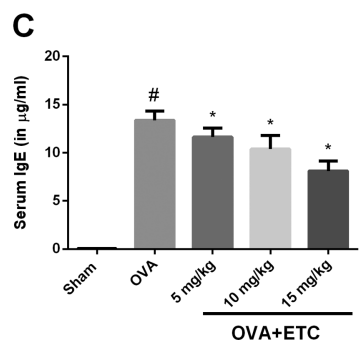

E

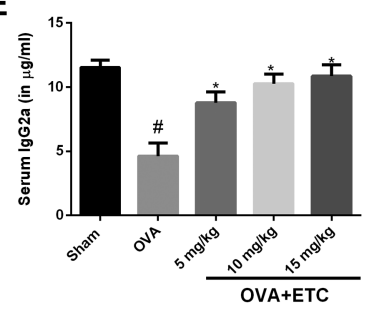

Figure 1. Effect of ETC on (A) nose-rub score and serum level of various biomarkers (B) histamine, (C) $\lg E$, (D) $\lg G_{1}$ and (E) $\lg \mathrm{g}_{2} \mathrm{a}$ in $A R$ mice. Data are presented as the mean \pm S.D. of three independent experiments. ${ }^{\#} P<0.05$ : significantly different from sham, ${ }^{*} P<0.05$ : significantly different from OVA-challenged mice.

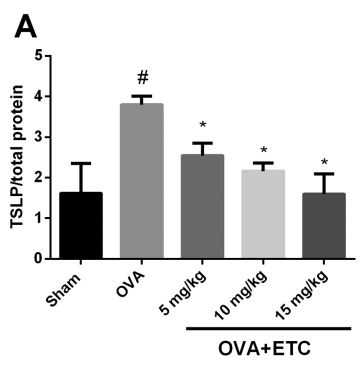

D

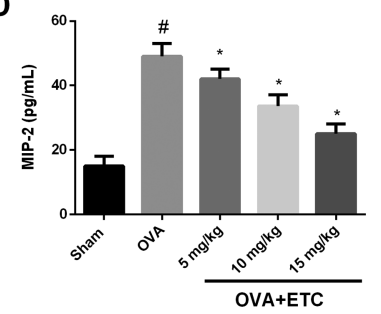

B

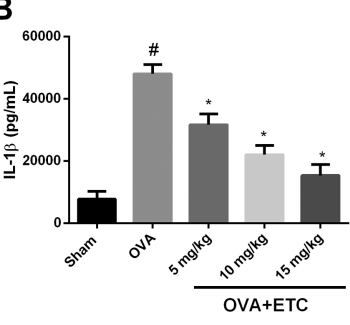

C

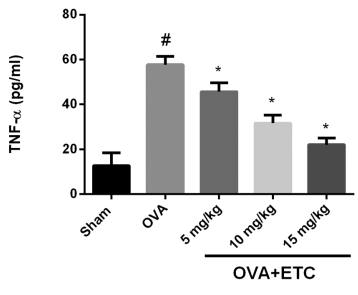

E

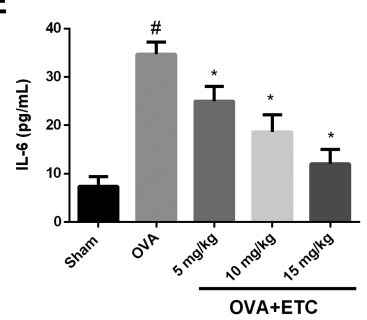

Figure 2. Effect of ETC on various inflammatory biomarkers in AR mice. (A) TSLP, (B) IL-1 $\beta$, (C) TNF- $a$, (D) MIP-2, and (E) IL-6. Data are presented as the mean \pm S.D. of three independent experiments. $\# P<0.05$ : significantly different from $P B S$ (control), ${ }^{*} P<0.05$ : significantly different from OVA-challenged mice.

possibly via modulation of serum immunoglobulins and indirectly also of histamine release.

\section{Effect of ETC on the levels of TSLP and inflammatory cytokines}

The release of various pro-inflammatory cytokines is a characteristic hallmark of AR following the exposure of allergens via inflammation. Thus, in Fig. $2 A$, the level of TSLP was found higher in OVA-treated mice as compared to sham. The level was found markedly reduced in ETC treated group in a dose-dependent manner as compared to sham. Serum IL-1 $\beta$ (Fig. 2B), TNF-a (Fig. $2 C$ ), and MIP-2 (Fig. 2D) were significantly increased in the OVA control group as compared with sham. Treatment with ETC causes a significant reduction in a dosedependent manner. We further investigated the ability of ETC to inhibit the production of IL-6, to understand its role in the last phase of AR. A significant rise in serum IL-6 was observed in the OVA control group indicating an inflammatory immune response. ETC decreased the IL-6 level in a dose-dependent manner. 

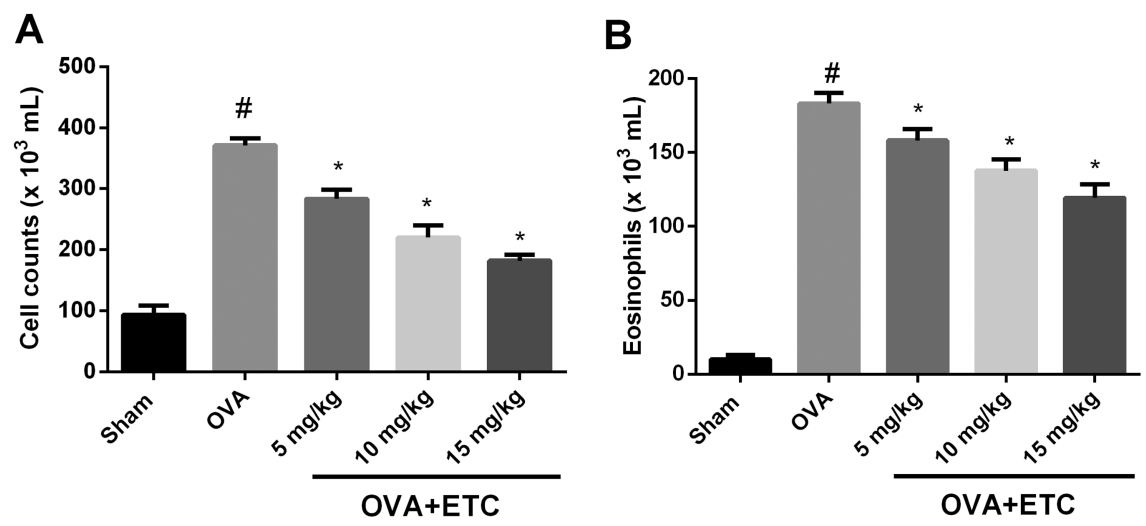

Figure 3. Effect of ETC on the (A) total cells and (B) eosinophil recruitment in AR mice. Data are presented as the mean \pm S.D. of three independent experiments. ${ }^{*} \mathrm{P}<0.05$ : significantly different from sham, ${ }^{*} \mathrm{P}<0.05$ : significantly different from OVA-challenged mice.

\section{Effect of ETC on the infiltration of total leukocytes and eosinophils into the airways in a murine model of allergic airway inflammation}

To determine whether ETC has effects on allergic airway inflammation, its effect was then determined on total cell infiltration and eosinophilia in the Broncho Alveolar Lavage Fluids (BALFs) of mice, Fig 3. In the sham group, the OVA challenge caused a marked increase of inflammatory cells in the BALF, especially eosinophils, which reflects the intensity of airway inflammation and the extent of cell infiltrates into the lungs, as compared to the sham-treated group. This was absent in the sham challenged negative control group. However, the number of total BAL cells was significantly decreased in the ETC-treated group in a dose-dependent manner in comparison to the OVAchallenged group (Fig. 3A). Moreover, the number of eosinophils in the BALF was also found reduced in ETC treated group (Fig. 3B). Our findings indicate that ETC reduces airway inflammation and prevents eosinophil infiltration into the airways in this allergy model.

\section{Histopathological examination of lung tissues after ETC administration}

To further examine the effects of ETC on lung pathology, histological analyses of lung sections were performed. As shown in Fig. 4, no apparent histological alteration was found in lung tissues of the sham-treated group. However, the OVA challenged mice showed marked lung injury suggested by a large number of infiltrations of inflammatory cells, lung tissue damage, and marked alveolar wall edema. These changes were significantly improved by treatment with the ETC in a dose-dependent manner.

\section{Effect of ETC on the TLR4/NF-KB signaling pathway and $\mathrm{COX}-2$ via western blot analysis}

In this part of the study, we try to understand the mechanism underlying the anti-inflammatory effect shown by ETC. Towards this, we have evaluated the effect of ETC on the expression of COX-2 and TLR4/ NF-KB signaling pathway and COX-2. As shown in Fig. 5, the level of both TLR-4 caspase-1, p- NF-KB $\mathrm{p} 65$, and $\mathrm{p}-\mathrm{I} \mathrm{kBa}$ were found significantly elevated in OVA-sensitized mice in comparison to sham. Upon administration of ETC, the levels of these tested proteins were found restored near to normal in a dosedependent manner. Moreover, the expression of COX-2 was also found reduced in ETC treated group in a dose-dependent manner.

\section{Docking analysis of ETC with COX-2 and NF-KB}

Prompted by the inhibitory activity of ETC against COX-2 and NF-KB, next, we intend to elaborate the critical receptor-ligand interactions by docking ETC into the active site of the 3D crystal structure of COX2 and NF-kB. This study provides the underlying basis for the inhibitory activity of ETC against COX-2 and NF-KB by enumerating the vital interactions responsible for its inhibitory activity. As shown in Fig. 6, the ETC was deeply engulfed and created numerous interactions with neighboring residues in the active site of COX-2 and NF-KB. Against COX-2, ETC created numerous pi-interactions with Val74, Arg106, Val102, Leu345, Leu517, Val 509, Tyr341, and Glu510. On the other hand, it also created pi-interactions with Lys 145, Cys59, and Thr143 in the active site of NF-KB. Thus, these results suggest that ETC might exert a significant anti-inflammatory effect in AR mice due to the strong 

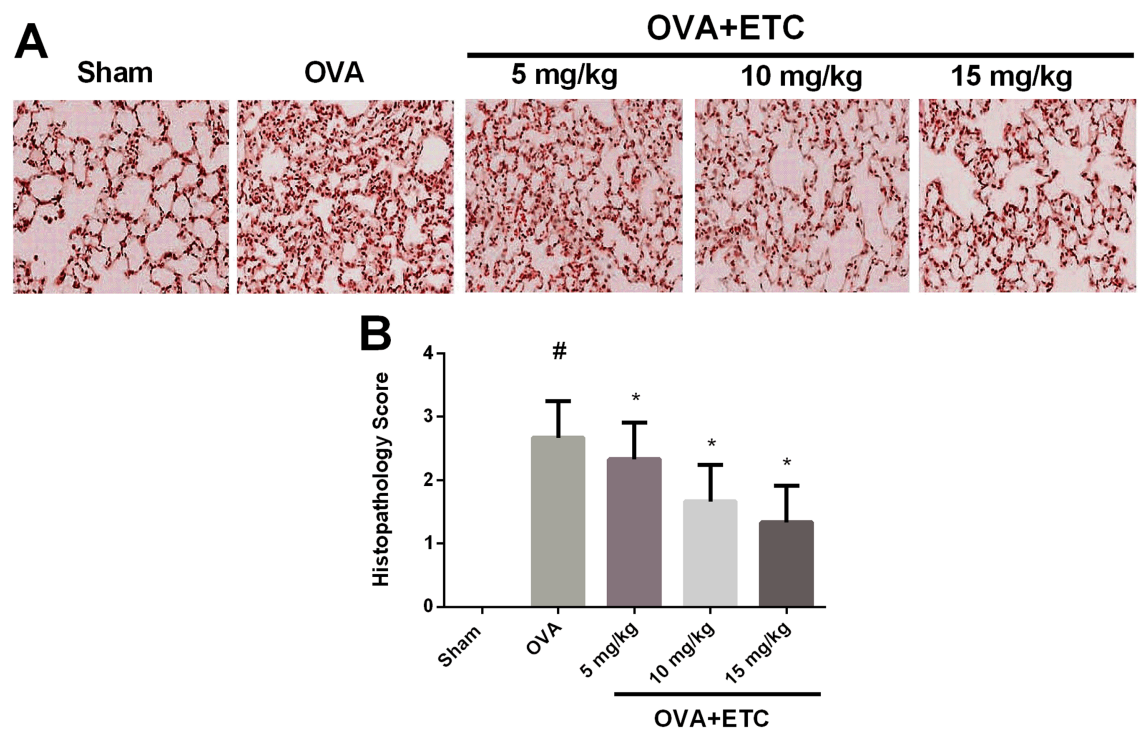

Figure 4. Effect of ETC on the (A) histopathology of lung tissues determined by $\mathrm{H}$ and $\mathrm{E}$ staining and representative histogram of (B) histopathology score. Data are presented as the mean \pm S.D. of three independent experiments. ${ }^{*} \mathrm{P}<0.05$ : significantly different from sham, ${ }^{*} \mathrm{P}<0.05$ : significantly different from OVA-challenged mice.
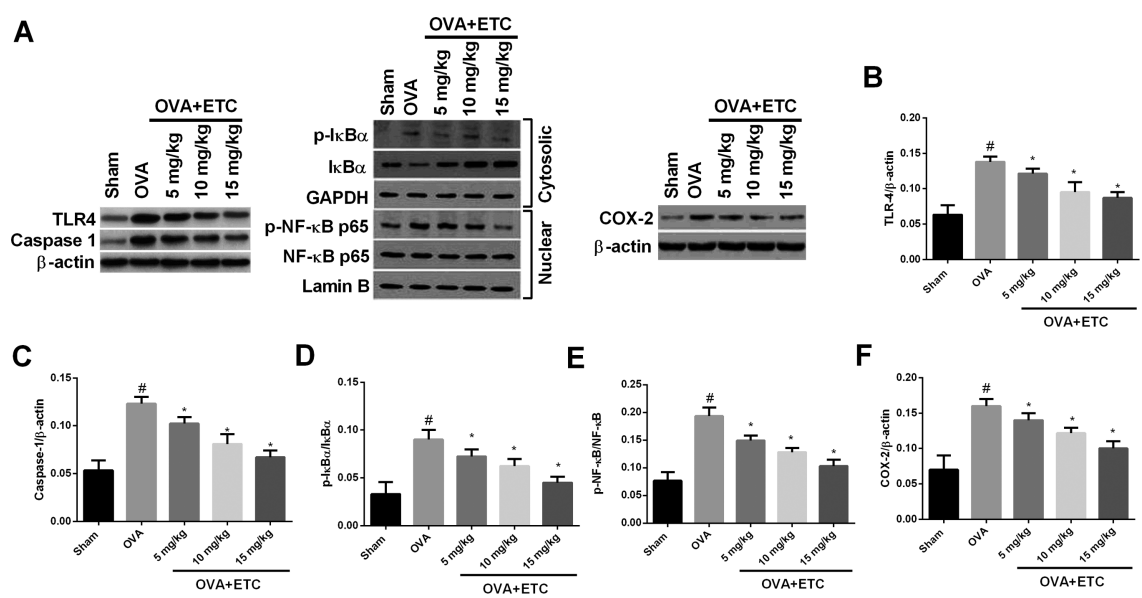

Figure 5. Effect of ETC on the expression of (A) COX-2 and NF-KB in AR determined by western blot and representative histogram of (B) COX-2 and $(C) N F-K B$. Data are presented as the mean \pm S.D. of three independent experiments. " $P<0.05$ : significantly different from sham, ${ }^{*} \mathrm{P}<0.05$ : significantly different from OVA-challenged mice.

inhibitory effect against COX-2 and NF-kB by making close-interatomic contacts into their active sites.

\section{Discussion}

In the present study, we have demonstrated that ellipticine protects mice from allergic rhinitis via attenuation of inflammation by inhibiting COX-2 and NF-KB. It also showed to reduce symptoms of $A R$ indicated by reduced frequency of nose-rubs and histopathological analysis of nasal mucosa via alleviating serum histamine level. The strong anti-inflammatory effect of ellipticine was attributable to its significant interaction with the active site of COX-2 and NF-KB. The murine model of AR in mice was induced by Ovalbumin which induces allergic reactions in mice similar to that of humans. It displayed characteristic allergic behaviors, such as increased sneezing and increased nose rubbing motions (Aswar et al., 2015; Zhang et al., 2015). Therefore, in the present study, the effect of ETC on various biomarkers was studied in the nasal mucosal tissues of mice. Ellipticine causes a reduction in noserubs in mice possibly via reducing the serum histamine level. It also causes a reduction of Th2-related antibodies $\lg E$ and $\lg G 1$ in serum together with up-regulation of Th1-related OVA-specific IgG2a which are known to 


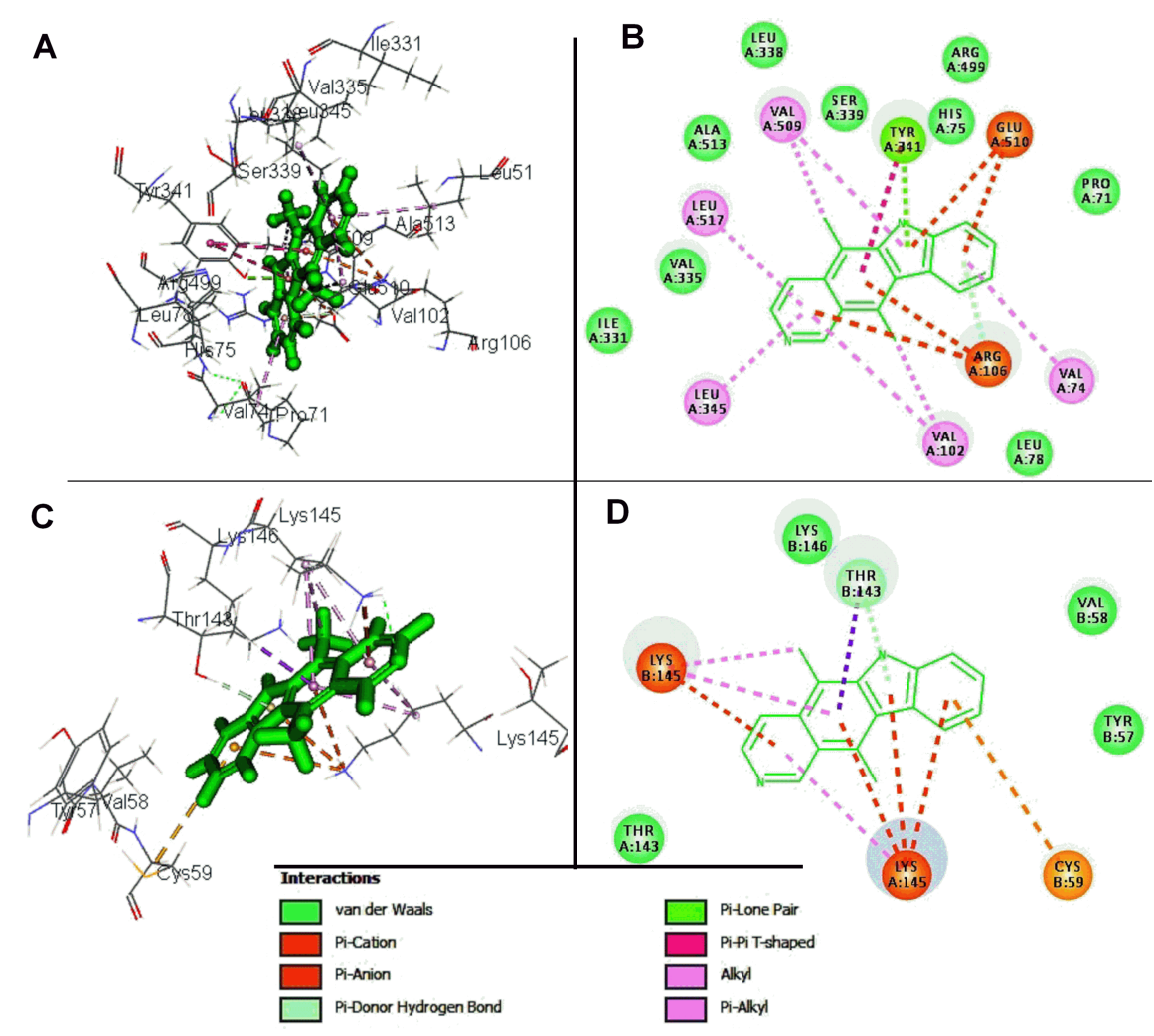

Figure 6. Docking analysis of ETC in COX-2 and NF-kB. (A) 3D-docked pose and (B) 2 D orientation in COX-2; (C) 3D-docked pose and (B) $2 \mathrm{D}$ orientation in NF-KB.

initiate hypersensitivity reaction in AR. Thus, it could be suggested that ETC might able to modulate the Th2 (humoral) immune response to protect AR. Various studies have shown a strong correlation between immunoglobulin and cytokines in AR (May and Fung, 2015; McDonald et al., 2015; Mlcek et al., 2016). Several studies have shown an elevated level of immunoglobulin's initiate recruitment of various pro-inflammatory cytokines which increases the permeability of eosinophils and total leukocyte counts (Pal et al., 2017; Tajiri et al., 2014).

Studies have shown that thymic stromal lymphopoietin (TSLP) has been reported as a treatment target for AR. TSLP is an epithelial cell-derived cytokine that plays a critical role in immune regulation in allergic conditions. TSLP is highly expressed in the nasal epithelia of patients with AR and activates and induces the release of various immune cells, such as IL-1 $\beta$, IL-6, IL-5, and TNF- $a$. It also increases eosinophils infiltration. TSLP-activated immune cells produce Th2 cytokines and chemokines and promote Th2-type immune reactions (Varricchi et al., 2018; Ziegler, 2012; Ziegler et al., 2013). A deficiency in TSLP receptors reduced allergic skin inflammation in ovalbumin (OVA)sensitized mice (Miyata et al., 2008). Recently, it was shown that human anti-TSLP monoclonal immunoglobulin treatment attenuated allergen-induced asthmatic responses in patients with mild allergic asthma (Gauvreau, O'Byrne, et al., 2014). In a meta-analysis of genome-wide association studies (GWAS) of atopic dermatitis or asthma patients, the single nucleotide polymorphism of TSLP, rs1837253, was associated with increased susceptibility for these diseases (Weidinger et al., 2013). Hence, TSLP strongly contributes to the pathogenesis of allergic diseases and is a molecular target for the treatment of these diseases (KlukowskaRötzler et al., 2013; Siracusa et al., 2013; Zhou et al., 2005; Ziegler, 2010). Thus, clinical trials to assess the efficacy of anti-IL-1 $\beta$, anti-IL-6, anti-TSLP, and anti-TNF-a drugs have been conducted about inflammatory disorders. In the present study, ellipticine reduces the level of TSLP, IL- $1 \beta$, TNF- $\alpha$, and MIP- 2 in the serum of AR mice. Thus, it could be suggested that ETC showed a significant effect against AR via reducing allergen sensitization through inhibition of TSLP. It also inhibited late inflammatory response in AR mice as indicated by a reduced level of IL- 6 . Eosinophils are bone marrowderived leukocytes demonstrated to have a causal role in AR pathogenesis including airway hyper-reactivity, elevated mucus production, and airway remodeling. 
It releases several cytotoxic proteins, such as major basic protein, platelet-activating factor, and leukotrienes, which are responsible for the characteristic damage of the airway epithelium (Choi et al., 2014; Eifan and Durham, 2016; Gauvreau, Harris, et al., 2014; Pal et al., 2017). In the present study, ellipticine cause dose-dependent reduction of eosinophils in BALF of AR mice. Our observation was further supported by histopathological examination of the lungs of ellipticine treated mice, which showed milder pathology, reduced inflammatory cell infiltrates, and a lower density of eosinophils in the lungs. NF-KB represents a family of inducible transcription factors, which after activation regulates a large array of genes involved in different processes of the immune and inflammatory responses. The NF-KB proteins are normally sequestered in the cytoplasm by a family of inhibitory proteins, such as IKB family members, and exist in dormant form. Upon activation, IKK phosphorylates IKBa at two $\mathrm{N}$-terminal serines and, thereby, triggers ubiquitin-dependent IKBa degradation in the proteasome, resulting in rapid and transient nuclear translocation of canonical NFKB members predominantly the p50/RelA and p50/cRel dimmers (Liu et al., 2017; Sun, 2017). Studies have shown that the TLR4/NF-KB signaling pathway plays a critical role in inflammatory cytokine production in AR. In the TLR4-mediated pathway, activated TLR4 increases phosphorylation of the downstream kinase, $p$-IKK $\beta$, which then induces IKBa phosphorylation, resulting in nuclear translocation of NF-KBp65 and regulation of immune/inflammatory responses (Covert et al., 2005; Pålsson-McDermott and O'Neill, 2004; Yao et al., 2013). In the present manuscript, we have shown that ETC could be able to inhibit the activation of NF-KB via inhibiting its nuclear translocation from the cytoplasm to the nucleus via decreasing the phosphorylation of $\mathrm{p}-\mathrm{I} \mathrm{kBa}$. The ETC suggested inhibiting NF-KB activation via inhibition of TLR-4 and caspase-1 (Fig. 5 B and C). Thus, it could be suggested that ETC might exert its anti-inflammatory effect against $A R$ in mice via inhibiting TLR4/NF-KB signaling pathway. Moreover, NF-KB also regulates the expression of several proteins such as pro-inflammatory cytokines, iNOS, and COX-2 (Kundu et al., 2006; Logan et al., 2007). COX-2 is a membranebound enzyme in the endoplasmic reticulum (ER). Its activity is induced by proinflammatory cytokines and produces prostaglandins that mediate the inflammatory response in the prostaglandin biosynthesis pathway. COX-2 is the key enzyme that catalyzes the conversion of arachidonic acid to PGE2. It is generally accepted that PGE2 is produced by COX-2 at sites of inflammation (Hawkey, 1999; Pasero and McCaffery, 2001). Therefore, it could be easily understood that the NF-KB transcription factor is uniquely positioned downstream of multiple innate and adaptive signaling pathways. They seem ideally placed to integrate and coordinate innate and adaptive signals required for the formation of productive immune responses. Thus, the effect of ETC was identified on the expression of COX2. Ellipticine showed a significant reduction in the level of COX-2 in AR mice as shown by western blot analysis (Fig. 5F). This result suggested that ETC might be able to inhibit the release of COX-2 via inhibiting the translocation of NF-KB. A body of evidence including clinical studies and animal models of AR has demonstrated significant involvement of COX-2 (Rahman et al., 2006) and NF-KB (Wee et al., 2017) in the progression of airway inflammation in AR. Therefore, it was envisaged that the ellipticine exert its anti-inflammatory effect against $A R$ in mice via strong inhibition of COX-2 and NF-KB activation. To understand the mechanism for this strong inhibition, ellipticine was allowed to dock into the active site of COX-2 and NF-KB. After observing $3 \mathrm{D}$ and $2 \mathrm{D}$ docked pose of ellipticine in the active site of both COX-2 and NF-KB, it has been found that, it created numerous vital inter-atomic contacts with the neighbouring residues which deemed responsible for its inhibitory activity. The interaction created by the ellipticine was found similar with the previously reported COX-2 (Bhat et al., 2018; El-Shoukrofy et al., 2019) and NF-kB inhibitor (Srivastava et al., 2015). Ellipticine exhibits toxic side effects, such as nausea and vomiting, hypertension, muscular cramp, fatigue which can be very pronounced, mouth dryness, and mycosis of the tongue and esophagus which limit its clinical use (Cros et al., 1987; Paoletti et al., 1980). It showed complete absence of hematological toxicity (Auclair, 1987). Concerning this, a recent study has successfully developed water soluble pro-drugs of ETC which are devoid of serious side-effects and may find significance in medical practice (De Oliveira et al., 2020).

\section{Conclusion}

The results of our finding demonstrated the significance of ellipticine in allergic rhinitis. Results suggested that ellipticine diminishes allergic inflammatory reactions and alleviates symptoms of AR by reducing 
the level of histamine and various pro-inflammatory cytokine via inhibition of COX-2 and NF-KB via inhibition of TLR4. These results imply that ellipticine could be used to control the allergic inflammatory response such as AR.

\section{Ethical approval}

Ethical approval to report this case was obtained from the institutional animal ethical committee of Xijing Hospital, China for biomedical experiments.

\section{Statement of human and animal rights}

All procedures in this study were conducted following the institutional animal ethical committee of Xijing Hospital, China (SPP/001/34) for biomedical experiments approved protocols.

\section{Statement of informed consent}

There are no human subjects in this article and informed consent is not applicable.

\section{Conflict of interest}

None.

\section{References}

Amin K (2012) The role of mast cells in allergic inflammation. Respiratory Medicine. DOI: 10.1016/j.rmed.2011.09.007.

Aswar U, Shintre S, Chepurwar S, et al. (2015) Antiallergic effect of piperine on ovalbumin-induced allergic rhinitis in mice. Pharmaceutical Biology 53(9): 1358-1366. DOI: 10.3109/13880209.2014.982299.

Auclair C (1987) Multimodal action of antitumor agents on DNA: The ellipticine series. Archives of Biochemistry and Biophysics 259(1): 1-14. DOI: 10.1016/0003-9861(87)90463-2.

Avincsal MO, Ozbal S, Ikiz AO, et al. (2014) Effects of topical intranasal doxycycline treatment in the rat allergic rhinitis model. Clinical and Experimental Otorhinolaryngology 7(2): 106-111. DOI: 10.3342/ceo.2014.7.2.106.

Bhat M, Gurubasavaraja Swamy PM, Poojary B, et al. (2018) Biological evaluation and in silico molecular docking study of a new series of thiazol-2-yl-hydrazone conglomerates. Research on Chemical Intermediates 44(4): 2779-2805. DOI: 10.1007/s11164-018-3261-z.

Bousquet J, Hellings PW, Agache I, et al. (2019) Allergic Rhinitis and its Impact on Asthma (ARIA) Phase 4 (2018): Change management in allergic rhinitis and asthma multimorbidity using mobile technology. Journal of Allergy and Clinical Immunology 143(3): 864-879. DOI: 10.1016/j.jaci.2018.08.049.
Brożek JL, Bousquet J, Agache I, et al. (2017) Allergic Rhinitis and its Impact on Asthma (ARIA) guidelines-2016 revision. Journal of Allergy and Clinical Immunology 140(4): 950-958. DOI: 10.1016/j.jaci.2017.03.050.

Chen DY, Chen YM, Chen HH, et al. (2011) Increasing levels of circulating Th17 cells and interleukin-17 in rheumatoid arthritis patients with an inadequate response to anti-TNF-a therapy. Arthritis Research and Therapy 13(4). DOI: 10.1186/ ar3431.

Choi CH, Poroyko V, Watanabe S, et al. (2014) Seasonal allergic rhinitis affects sinonasal microbiota. American Journal of Rhinology and Allergy 28(4): 281-286. DOI: 10.2500/ ajra.2014.28.4050.

Covert MW, Leung TH, Gaston JE, et al. (2005) Achieving stability of lipopolysaccharide-induced NF-KB activation. Science 309(5742): 1854-1857. DOI: 10.1126/science.1112304.

Cros S, Pepin O, Colas C, et al. (1987) Physicochemical And Pharmacological Properties Of The Antitumor Ellipticine Derivative 2-(Diethylamino-2-Ethyl)9-Hydroxy Ellipticinium-Chloride, Hcl. Cancer Research 47(23): 6254-6261.

De Oliveira RC, Pontes GS, Kostyuk A, et al. (2020) Anticancer and immunomodulatory activities of a novel water-soluble derivative of ellipticine. Molecules 25(9). DOI: 10.3390/ molecules25092130.

Eifan AO and Durham SR (2016) Pathogenesis of rhinitis. Clinical and Experimental Allergy. DOI: 10.1111/cea.12780.

El-Shoukrofy MS, Abd El Razik HA, AboulWafa OM, et al. (2019) Pyrazoles containing thiophene, thienopyrimidine and thienotriazolopyrimidine as COX-2 selective inhibitors: Design, synthesis, in vivo anti-inflammatory activity, docking and in silico chemo-informatic studies. Bioorganic Chemistry 85: 541-557. DOI: 10.1016/j.bioorg.2019.02.036.

Gauvreau GM, Harris JM, Boulet LP, et al. (2014) Targeting membrane-expressed IgE B cell receptor with an antibody to the M1 prime epitope reduces IgE production. Science Translational Medicine 6(243). DOI: 10.1126/scitranslmed.3008961.

Gauvreau GM, O'Byrne PM, Boulet L-P, et al. (2014) Effects of an Anti-TSLP Antibody on Allergen-Induced Asthmatic Responses. New England Journal of Medicine 370(22): 21022110. DOI: 10.1056/nejmoa1402895.

Han NR, Moon PD, Nam SY, et al. (2016) Inhibitory effects of atractylone on mast cell-mediated allergic reactions. Chemico-Biological Interactions 258: 59-68. DOI: 10.1016/j. cbi.2016.08.015.

Hawkey CJ (1999) COX-2 inhibitors. Lancet. DOI: 10.1016/ S0140-6736(98)12154-2.

Incorvaia C, Cavaliere C, Frati F, et al. (2018) Allergic rhinitis. Journal of Biological Regulators and Homeostatic Agents 32(1): 61-66. DOI: 10.21518/2079-701x-2018-20-76-79.

Kamm JL, Nixon AJ and Witte TH (2010) Cytokine and catabolic enzyme expression in synovium, synovial fluid and articular cartilage of naturally osteoarthriticequine carpi. Equine Veterinary Journal 42(8): 693-699. DOI: 10.1111/ j.2042-3306.2010.00140.x.

Klukowska-Rötzler J, Chervet L, Müller EJ, et al. (2013) Expression of thymic stromal lymphopoietin in canine atopic dermatitis. Veterinary Dermatology 24(1). DOI: 10.1111/ j.1365-3164.2012.01096.x. 
Kundu JK, Shin YK, Kim SH, et al. (2006) Resveratrol inhibits phorbol ester-induced expression of COX-2 and activation of NF-KB in mouse skin by blocking IкB kinase activity. Carcinogenesis 27(7): 1465-1474. DOI: 10.1093/carcin/bgi349.

Li X, Ye C, Mulati M, et al. (2020) Ellipticine blocks synergistic effects of IL-17A and TNF- $a$ in epithelial cells and alleviates severe acute pancreatitis-associated acute lung injury. Biochemical Pharmacology 177: 113992. DOI: 10.1016/j. bcp.2020.113992.

Liu T, Zhang L, Joo D, et al. (2017) NF-KB signaling in inflammation. Signal Transduction and Targeted Therapy 2: 17023. DOI: 10.1038/sigtrans.2017.23.

Logan RM, Gibson RJ, Sonis ST, et al. (2007) Nuclear factor-KB (NF-KB) and cyclooxygenase-2 (COX-2) expression in the oral mucosa following cancer chemotherapy. Oral Oncology 43(4): 395-401. DOI: 10.1016/j.oraloncology.2006.04.011.

Masood F, Chen P, Yasin T, et al. (2013) Encapsulation of Ellipticine in poly-(3-hydroxybutyrate-co-3- hydroxyvalerate) based nanoparticles and its in vitro application. Materials Science and Engineering C 33(3): 1054-1060. DOI: 10.1016/j. msec.2012.11.025.

May RD and Fung M (2015) Strategies targeting the IL-4/IL13 axes in disease. Cytokine 75(1): 89-116. DOI: 10.1016/j. cyto.2015.05.018.

McDonald JL, Cripps AW and Smith PK (2015) Mediators, Receptors, and Signalling Pathways in the Anti-Inflammatory and Antihyperalgesic Effects of Acupuncture. Evidencebased Complementary and Alternative Medicine. DOI: 10.1155/2015/975632.

Miyata M, Hatsushika K, Ando T, et al. (2008) Mast cell regulation of epithelial TSLP expression plays an important role in the development of allergic rhinitis. European Journal of Immunology 38(6): 1487-1492. DOI: 10.1002/eji.200737809.

Mlcek J, Jurikova T, Skrovankova S, et al. (2016) Quercetin and its anti-allergic immune response. Molecules. DOI: 10.3390/ molecules21050623.

Newman DJ and Cragg GM (2016) Natural Products as Sources of New Drugs from 1981 to 2014. Journal of Natural Products. DOI: 10.1021/acs.jnatprod.5b01055.

Okubo K, Kurono Y, Ichimura K, et al. (2017) Japanese guidelines for allergic rhinitis 2017. Allergology International. DOI: 10.1016/j.alit.2016.11.001.

Pal I, Babu AS, Halder I, et al. (2017) Nasal smear eosinophils and allergic rhinitis. Ear, Nose and Throat Journal 96(10-11): E17E22. DOI: 10.1177/0145561317096010-1105.

Pålsson-McDermott EM and O'Neill LAJ (2004) Signal transduction by the lipopolysaccharide receptor, Toll-like receptor-4. Immunology. DOI: 10.1111/j.1365-2567.2004.01976.x.

Pandrangi SL, Chikati R, Chauhan PS, et al. (2014) Effects of ellipticine on ALDH1A1-expressing breast cancer stem cells-An in vitro and in silico study. Tumor Biology 35(1): 723-737. DOI: 10.1007/s13277-013-1099-y.

Paoletti C, Le Pecq JB, Dat-Xuong N, et al. (1980) Antitumor activity, pharmacology, and toxicity of ellipticines, ellipticinium, and 9-hydroxy derivatives: preliminary clinical trials of 2-methyl-9-hydroxy ellipticinium (NSC 264-137). Recent results in cancer research. Fortschritte der Krebsforsc- hung. Progrès dans les recherches sur le cancer. DOI: 10.1007/ 978-3-642-81488-4_15.

Pasero C and McCaffery M (2001) Selective COX-2 inhibitors. American Journal of Nursing 101(4). DOI: 10.1097/ 00000446-200104000-00024.

Patridge E, Gareiss P, Kinch MS, et al. (2016) An analysis of FDAapproved drugs: Natural products and their derivatives. Drug Discovery Today. DOI: 10.1016/j.drudis.2015.01.009.

Rahman A, Yatsuzuka R, Jiang S, et al. (2006) Involvement of cyclooxygenase-2 in allergic nasal inflammation in rats. International immunopharmacology 6(11): 1736-1742. DOI: 10.1016/j.intimp.2006.07.015.

Seidman MD, Gurgel RK, Lin SY, et al. (2015) Clinical Practice Guideline: Allergic Rhinitis. Otolaryngology-Head and Neck Surgery 152: S1-S43. DOI: 10.1177/0194599814561600.

Siracusa MC, Saenz SA, Tait Wojno ED, et al. (2013) Thymic Stromal Lymphopoietin-Mediated Extramedullary Hematopoiesis Promotes Allergic Inflammation. Immunity 39(6): 11581170. DOI: 10.1016/j.immuni.2013.09.016.

Small P, Keith PK and Kim H (2018) Allergic rhinitis. Allergy, Asthma and Clinical Immunology. DOI: 10.1186/s13223-018-0280-7.

Srivastava JK, Awatade NT, Bhat HR, et al. (2015) Pharmacological evaluation of hybrid thiazolidin-4-one-1,3,5-triazines for NF-KB, biofilm and CFTR activity. RSC Advances 5(108): 88710-88718. DOI: 10.1039/c5ra09250g.

Stiborova M and Frei E (2014) Ellipticines as DNA-Targeted Chemotherapeutics. Current Medicinal Chemistry 21(5): 575-591. DOI: 10.2174/09298673113206660272.

Sun SC (2017) The non-canonical NF-KB pathway in immunity and inflammation. Nature Reviews Immunology. DOI: 10.1038/nri.2017.52.

Tajiri T, Niimi A, Matsumoto H, et al. (2014) Prevalence and clinical relevance of allergic rhinitis in patients with classic asthma and cough variant asthma. Respiration 87(3): 211-218. DOI: 10.1159/000355706.

Tao S, Meng S, Zheng X, et al. (2017) ATM participates in the regulation of viability and cell cycle via ellipticine in bladder cancer. Molecular Medicine Reports 15(3): 1143-1148. DOI: 10.3892/mmr.2017.6141.

Tian LX, Li XY, Tang X, et al. (2020) Ellipticine Conveys Protective Effects to Lipopolysaccharide-Activated Macrophages by Targeting the JNK/AP-1 Signaling Pathway. Inflammation 43(1). Inflammation: 231-240. DOI: 10.1007/s10753-019-01112-z.

Varricchi G, Pecoraro A, Marone Giancarlo, et al. (2018) Thymic stromal lymphopoietin isoforms, inflammatory disorders, and cancer. Frontiers in Immunology. DOI: 10.3389/ fimmu.2018.01595.

Wee JH, Zhang YL, Rhee CS, et al. (2017) Inhibition of allergic response by intranasal selective NF-KB decoy oligodeoxynucleotides in a murine model of allergic rhinitis. Allergy, Asthma and Immunology Research 9(1): 61-69. DOI: 10.4168/ aair.2017.9.1.61.

Weidinger S, Willis-Owen SAG, Kamatani Y, et al. (2013) A genome-wide association study of atopic dermatitis identifies loci with overlapping effects on asthma and psoriasis. Human Molecular Genetics 22(23): 4841-4856. DOI: 10.1093/ hmg/ddt317. 
Yao L, Kan EM, Lu J, et al. (2013) Toll-like receptor 4 mediates microglial activation and production of inflammatory mediators in neonatal rat brain following hypoxia: Role of TLR4 in hypoxic microglia. Journal of Neuroinflammation 10. DOI: 10.1186/1742-2094-10-23.

Yasar M, Savranlar Y, Karaman H, et al. (2016) Effects of propolis in an experimental rat model of allergic rhinitis. American Journal of Otolaryngology - Head and Neck Medicine and Surgery 37(4): 287-293. DOI: 10.1016/j.amjoto.2016.03.007.

Zhang N, Li H, Jia J, et al. (2015) Anti-inflammatory effect of curcumin on mast cell-mediated allergic responses in ovalbumin-induced allergic rhinitis mouse. Cellular Immunology 298(1-2): 88-95. DOI: 10.1016/j.cellimm.2015.09.010.

Zhou B, Comeau MR, De Smedt T, et al. (2005) Thymic stromal lymphopoietin as a key initiator of allergic airway inflammation in mice. Nature Immunology 6(10): 1047-1053. DOI: 10.1038/ni1247.
Ziegler SF (2010) The role of thymic stromal lymphopoietin (TSLP) in allergic disorders. Current Opinion in Immunology. DOI: 10.1016/j.coi.2010.10.020.

Ziegler SF (2012) Thymic stromal lymphopoietin and allergic disease. Journal of Allergy and Clinical Immunology. DOI: 10.1016/j.jaci.2012.07.010.

Ziegler SF, Roan F, Bell BD, et al. (2013) The Biology of Thymic Stromal Lymphopoietin (TSLP). In: Advances in Pharmacology, pp. 129-155. DOI: 10.1016/B978-0-12-404717-4.00004-4.

Zissler UM, Esser-Von Bieren J, Jakwerth CA, et al. (2016) Current and future biomarkers in allergic asthma. Allergy: European Journal of Allergy and Clinical Immunology. DOI: 10.1111/ all.12828. 